\title{
Opto-box: Optical modules and mini-crate for ATLAS pixel and IBL detectors
}

\author{
David Bertsche ${ }^{1, a}$, on behalf of the ATLAS Collaboration \\ ${ }^{1}$ University of Oklahoma, United States
}

\begin{abstract}
The opto-box is a custom mini-crate for housing optical modules which process and transfer optoelectronic data. Many novel solutions were developed for the custom design and manufacturing. The system tightly integrates electrical, mechanical, and thermal functionality into a small package of size $35 \times 10 \times 8 \mathrm{~cm}^{3}$. Special attention was given to ensure proper shielding, grounding, cooling, high reliability, and environmental tolerance. The custom modules, which incorporate Application Specific Integrated Circuits, were developed through a cycle of rigorous testing and redesign. In total, fourteen opto-boxes have been installed and loaded with modules on the ATLAS detector. They are currently in operation as part of the LHC run 2 data read-out chain. This conference proceeding is in support of the poster presented at the International Conference on New Frontiers in Physics (ICNFP) 2015 [1].
\end{abstract}

\section{Introduction}

ATLAS is a general-purpose particle detector located at the Large Hadron Collider (LHC) at CERN [2]. The Pixel and IBL detectors are the innermost systems which provide crucial tracking and vertexing functions. These detectors produce electronic signals which must be converted to optical signals for read-out. The opto-box is a custom mini-crate for housing the optoelectronic signal transceiver modules (opto-boards) which process and transfer this optoelectronic data.

During the first LHC long shut down (LS1) the opto-boards were moved to a new location outside of the Inner Detector. This decision was motivated by past pixel module failures in order to increase ease of access to the system during future data taking runs. This system was required to fit into the available space, so the opto-box was designed and constructed for this purpose. New high reliability opto-boards (including Application Specific Integrated Circuits, ASICs) were also developed and produced. In addition, this system was implemented to support the new Insertable B-Layer (IBL) of pixel modules also installed during LS1. The full system requirements and specifications are available in the engineering documentation [3].

\section{Design}

Each opto-box is of size $35 \times 10 \times 8 \mathrm{~cm}^{3}$, having 30 wire bundles, 72 fibre cables, and two cooling pipes connected. For ease of installation and maintenance, much consideration was given to cable management and physical integration with the already existing detector components.

The opto-box is required to house, cool, and power the opto-boards, one of which is shown in Figure 1. An important consideration in the design was the integration of electrical, mechanical, and thermal systems. Several novel solutions were developed to meet these specific requirements.

ae-mail: david.bertsche@cern.ch 


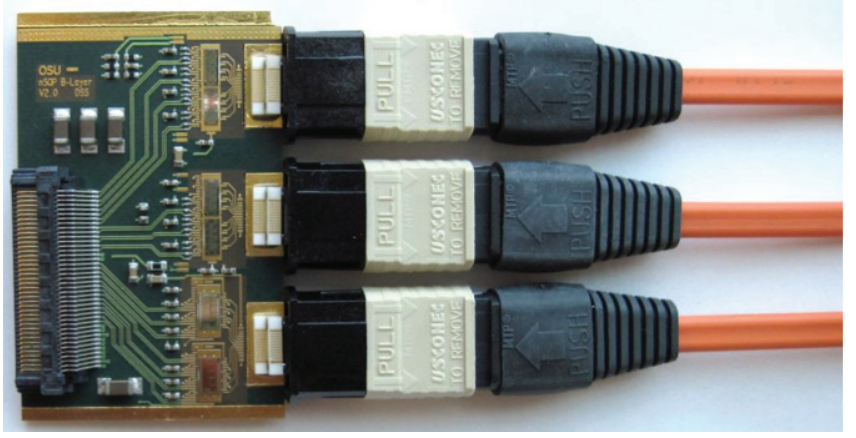

Figure 1: Opto-boards are optoelectronic signal transceiver modules that convert signals between electrical and optical form by using vertical-cavity surface-emitting-lasers (VCSELs). Optical fibers carry signals between the opto-boards and the read-out electronics.

The opto-box was designed as a micro-crate, with a slot and spring mechanism that allows insertion of the opto-boards from one side. A detailed mechanical design drawing of the opto-box is shown in Figure 2, where all the components shown provide mechanical support. In addition, some components also provide other functions. For example, the motherboard Printed Circuit Board (PCB) on the bottom distributes power and control signals to the opto-boards and the cooling rail at the top carries away excess heat generated by the boards.

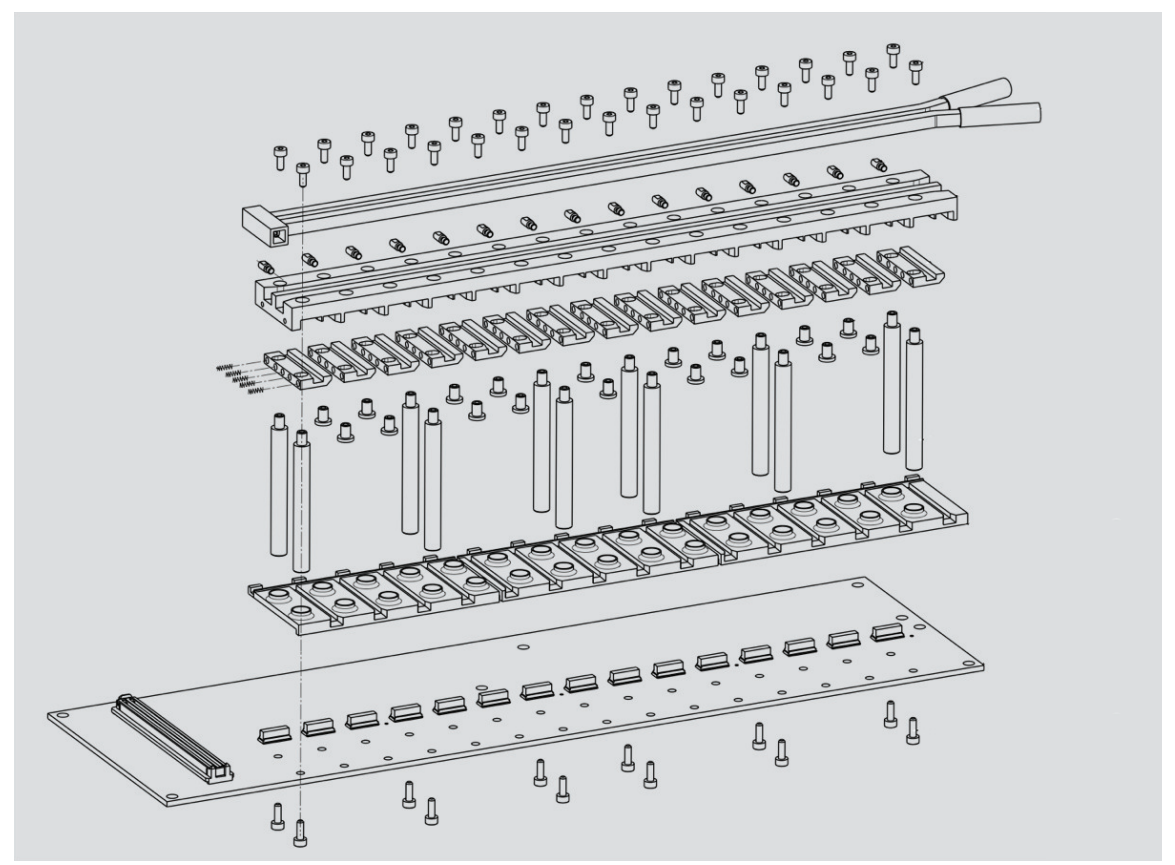

Figure 2: Computer Aided Design (CAD) image of the opto-box frame. 
Cooling is provided by means of thermal contact with an aluminium cooling rail embedded with copper pipes through which $\mathrm{C}_{6} \mathrm{~F}_{14}$ fluid is chilled to $16^{\circ} \mathrm{C}$ is circulated by the ATLAS monophase cooling system. This fluorocarbon is chosen because of its inert and radiation-hard properties. The expected maximum power usage per opto-board is $1685 \mathrm{~mW}$ and $2385 \mathrm{~mW}$ for Pixel and IBL boards respectively.

New pixel electrical readout (ER) bundle connectors were developed, as shown in Figure 3. They contain an integrated PCB and micro-connectors which enables the connection of the ER bundle, the opto-board and the opto-box using only one connector.

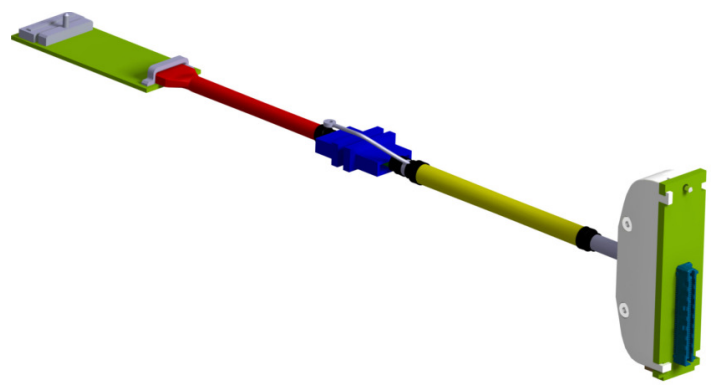

Figure 3: Computer Aided Design (CAD) image of the electrical readout cables used to attach each of the opto-boards to the detector. 24 Electrical Readout (ER) bundles carry electrical signals between the opto-box and the Pixel and IBL Detectors. 24 connector boards connect the ER bundles, the motherboard and the opto-boards.

Figure 4 shows a CAD drawing of the entire opto-box design, complete with all components. In total each opto-box integrates two PCBs (each with 300 conductive traces across 8 layers), four types of connectors, and 2284 electrical connections. Two versions of the opto-box were designed, one with 15 slots for the IBL detector, and one with 24 slots for the Pixel detector. In fact, two mirror image versions of the Pixel opto-box were also designed. This allows the Pixel opto-boxes to be installed back-to-back in pairs. There are two IBL and twelve Pixel opto-boxes installed on the ATLAS Detector, for a total of fourteen.

\section{Validation}

Reliability was built into the opto-box system. Electromagnetic shielding continuity is provided by using shielded connectors, PCB edge plating and PCB copper pours. High quality parts were used in the construction, including custom machined metal structures, IPC class 3 PCBs and aerospace grade connectors.

Many tests were performed at all stages of the fabrication and installation process. Electrical continuity of each PCB was verified at the PCB fabrication facility prior to delivery to CERN. The PCB boards were assembled with the connectors at the CERN PCB assembly facility. The assembled boards were again tested with a custom made test apparatus. Two of the PCB boards were found to be defective and had to be reassembled.

A custom made thermal test apparatus was used to ensure that no heat load is dissipated into surrounding detector systems. A prototype opto-box was loaded with dummy boards that used resistors to simulate heat loads. Temperatures were measured at various points using thermistors and the data 


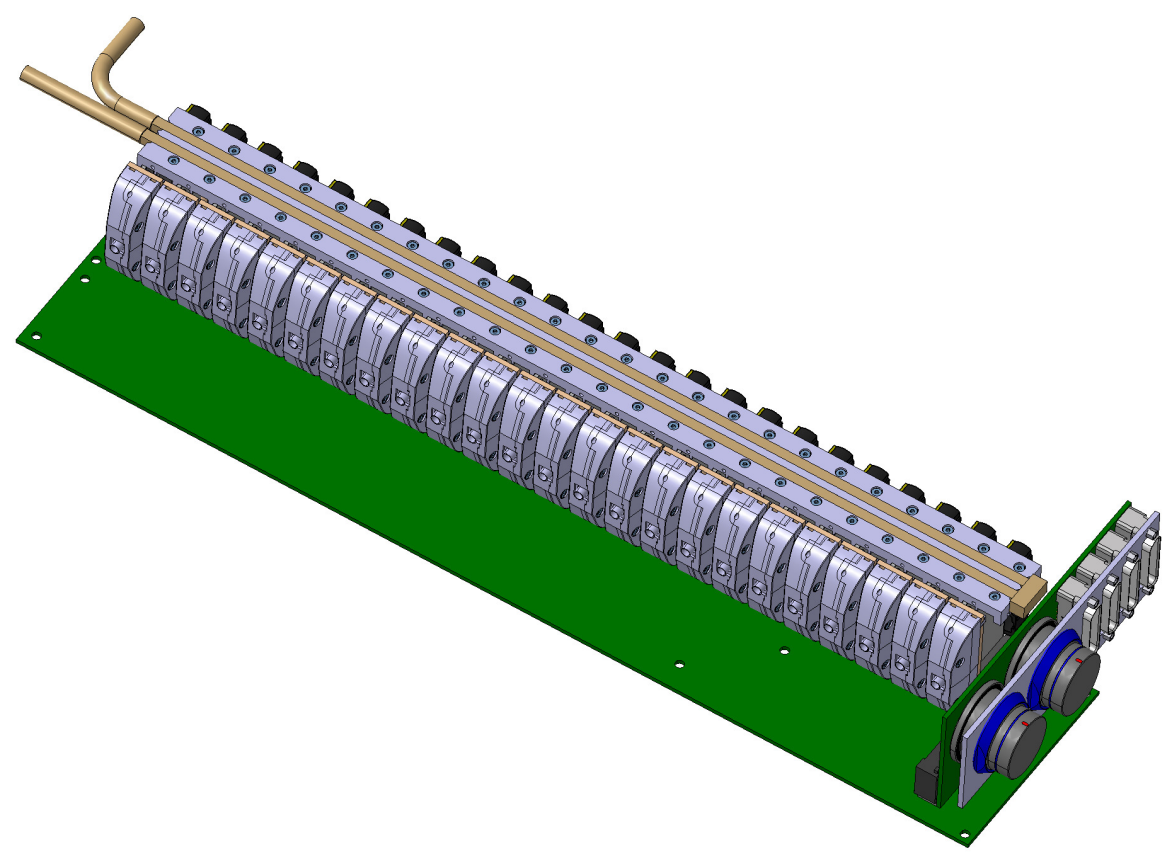

Figure 4: Computer Aided Design (CAD) image of the opto-box design for the Pixel Detector. The opto-boxes for the IBL Detector have fewer opto-board slots, but otherwise identical. The cooling pipe carries away heat using $\mathrm{C}_{6} \mathrm{~F}_{14}$ fluid at $16^{\circ} \mathrm{C}$. The motherboard distributes power and control signals to the opto-boards. The end board transfers power and control signals from six cables into the motherboard.

was read out using a custom-written MATLAB program. This test verified that the cooling system was adequate.

The opto-board design was iterated until it passed rigorous testing at humidity and temperature levels far above expected operating conditions. The cooling pipes were pressure tested before and after installation to ensure that there were no leaks.

After assembly and installation all the components of each opto-box were again tested and validated for use. PCB coupons have been reserved for accelerated age studies, which will be used to forestall future failures.

\section{Installation}

The opto-boxes are installed on each end of barrel section of the LAr electromagnetic calorimeter, shown in Figure 5. They are still deep inside ATLAS, just outside the Inner Detector system, and can only be accessed when the end caps are removed. But this is still more accessible than their previous location and increases serviceability. Figure 6 shows one pair of the opto-boxes as installed in the ATLAS Detector.

Custom tooling was developed for installing in the opto-boards to avoid damage and to gain enough access within limited space. In addition, since the opto-boxes are installed side-by-side, 


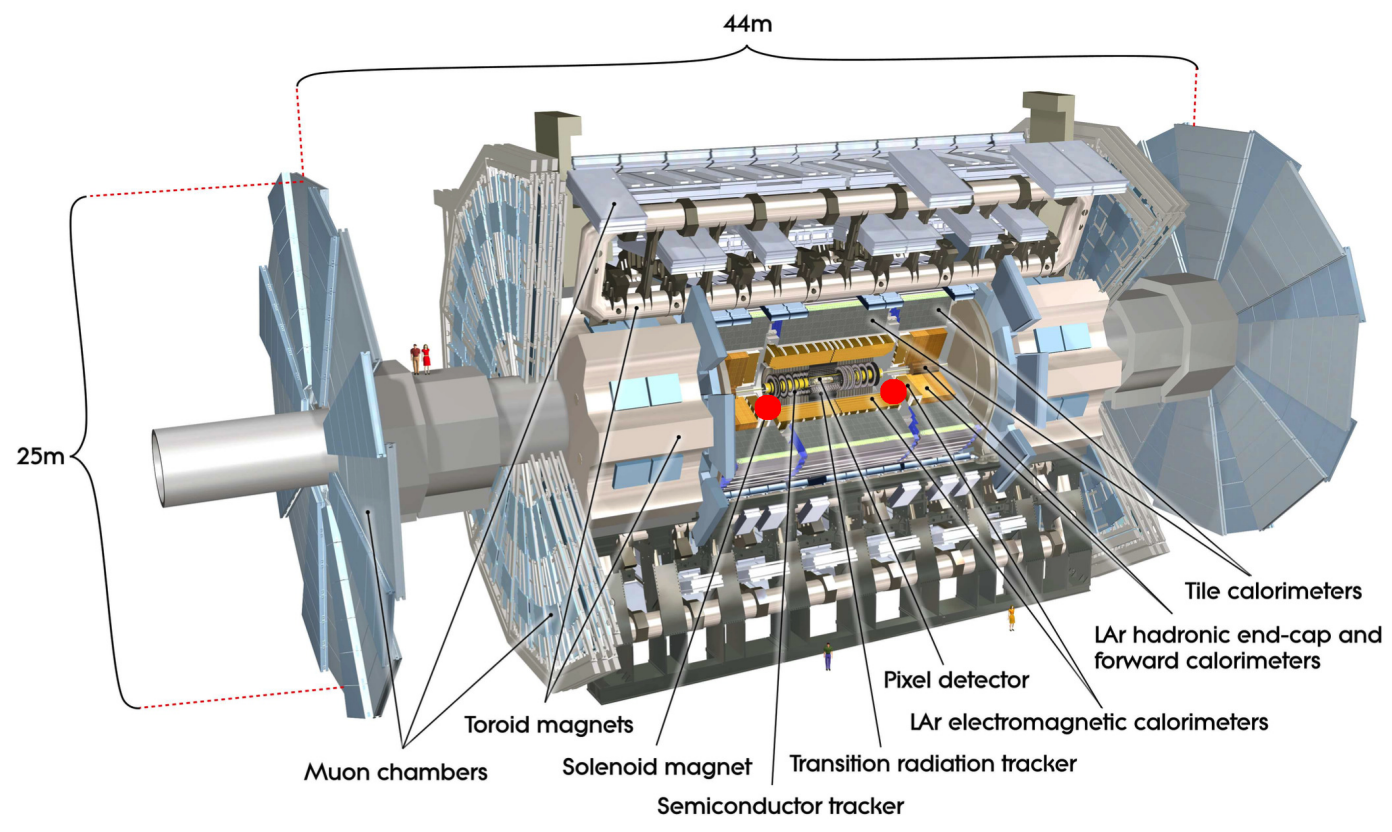

Figure 5: The opto-boxes are installed symmetrically on both sides of the ATLAS Detector. Their approximate locations are indicated by the red dots. They are installed on each end of barrel section of the LAr electromagnetic calorimeter, which is colored yellow in this figure.

mirror image Pixel Detector versions were made in order to facilitate easier access for the delicate opto-boards.

\section{Operation}

The opto-boxes and opto-boards began use in 2015 as ATLAS started to take run 2 data and are part of the opto-electronic readout chain. Figures 7 and 8 show that the cooling system and power systems are functional and operating within the expected parameters. 


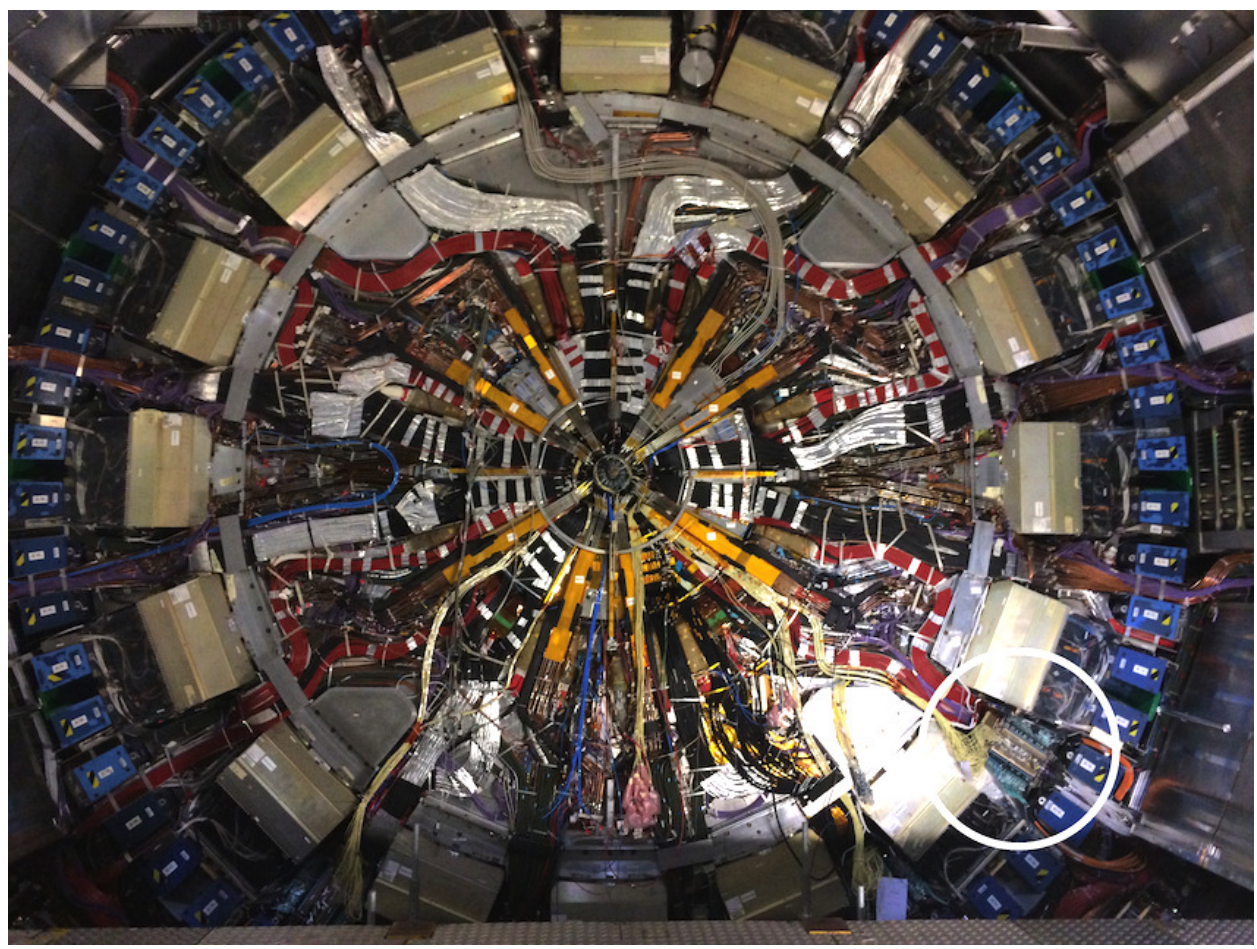

Figure 6: The white circle shows one Pixel opto-box pair installation location on the ATLAS Detector when it was still open during LS1.

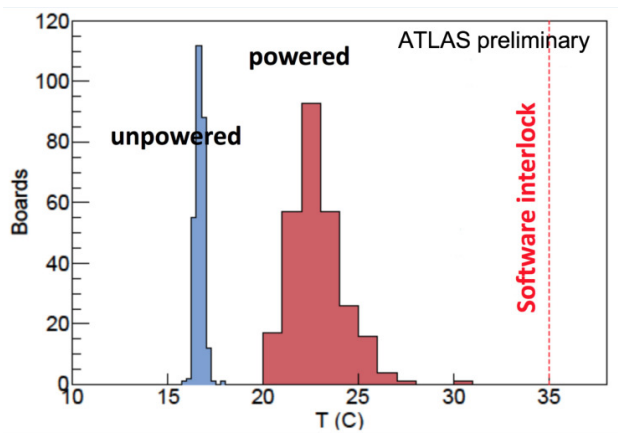

(a)

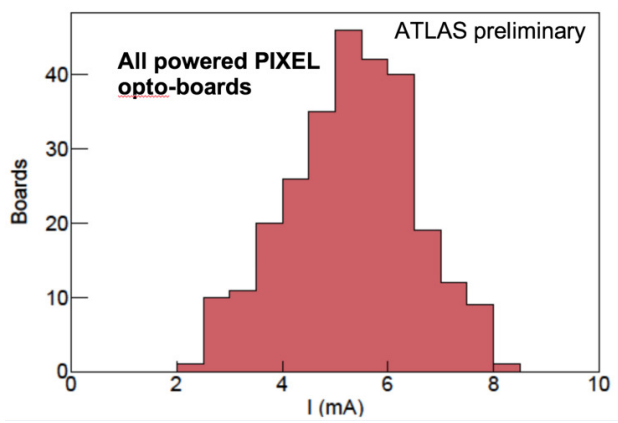

(b)

Figure 7: After installation, the 272 operational Pixel opto-boards are all adequately cooled below the $35^{\circ} \mathrm{C}$ threshold (a). They all receive adequate current $(>0.1 \mathrm{~mA})$ to ensure the required bit error rate of $<10^{-9}$ (b). 


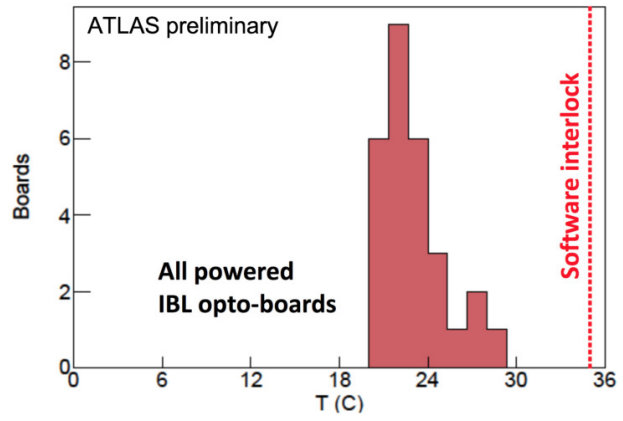

(a)

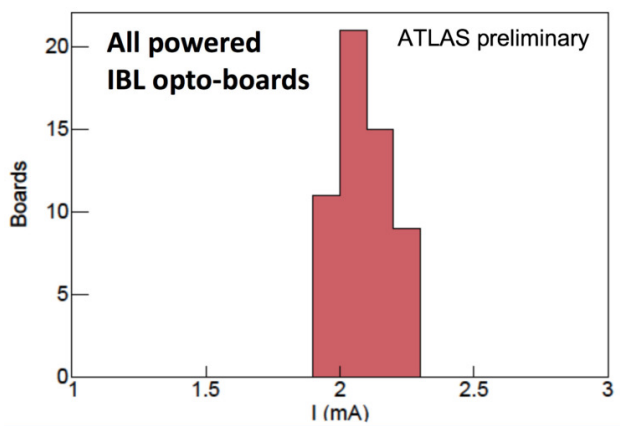

(b)

Figure 8: After installation, the 28 operational IBL opto-boards are all adequately cooled below the $35^{\circ} \mathrm{C}$ threshold (a). They all receive adequate current $(>0.1 \mathrm{~mA})$ to ensure the required bit error rate of $<10^{-9}$.

\section{Summary}

The opto-boxes provide ATLAS Pixel and IBL Detectors with reliable, tightly integrated, and serviceable mini-crates and modules for the optoelectronic data transfer system. They are currently operational as an integral part of data collection for LHC run 2.

\section{References}

1. “Opto-Box”, Poster, ATL-INDET-SLIDE-2015-461, https://cdsweb.cern.ch/record/2045532

2. "The ATLAS Experiment at the CERN Large Hadron Collider", ATLAS Collaboration, 2008 JINST 3 S08003

3. "Engineering specification for the Pixel services after the nSQPs installation", ATLAS Project Document, ATL-IP-ES-0148, https://edms.cern.ch/document/1101163/4 